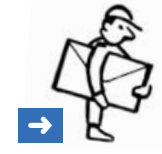

\section{Mises à jour dispendieuses du TARMED}

Mon époux, qui est médecin de famille, gère un cabinet à la campagne. Comme son temps est entièrement occupé par le traitement des patients et les travaux administratifs qui s'y rapportent, l'aide que je lui fournis comme collaboratrice (infirmière AKP, puis DN II, actuellement spécialiste en soins infirmiers) au cabinet médical est d'autant plus importante; je collabore aussi, par exemple, à la mise à jour du TARMED 1.05. Nous avons appris depuis longtemps à appliquer le TARMED en suivant son évolution; nous sommes devenus de véritables professionnels en la matière.

$\mathrm{Au}$ fond, nous sommes très contents de l'organisation de notre cabinet, car nous sommes à niveau. Tout fonctionne: traitements, décomptes, facturation. Une seule chose nous dérange ... les corrections fréquentes du TARMED.

Les modifications, petites en soi, de la version 1.05 , tel que le passage des positions de radiologie de 30.xxxx à 39.xxxx, sont très dispendieuses.

A fin mars 2007, la mise à jour nous a coûté Fr. 1459.05. Le 10 janvier 2008, nous avons dû à nouveau payer Fr. 1018.95 pour des modifications qui étaient minimes pour le praticien généraliste. Certes, nous faisons toutes les mises à jour nous-mêmes. Ces montants sont de pures taxes de licence. L'entreprise de logiciels n'a certainement pas la tâche facile. Les délais incorrectement courts avec $\mathrm{x}$ dates de report sur les jours fériés ne causent que des dérangements. S'y ajoute encore la publication erronée de la position 39.2100 au lieu de 39.2000. Pour le BMS ou santésuisse, c'est simple et rapide à corriger au moyen d'un erratum. Pour ma part, j'ai dû réfléchir longtemps pour savoir où se trouvait la faute, vu que la firme de logiciel ne livre que le chiffre 39.2000 comme position.
D'ailleurs, nous sommes à notre cabinet médical aussi en mesure de faire des changements de pansement de $10,1 \mathrm{~cm}$ à $20 \mathrm{~cm}$ ou même plus grand. Mais cette position n'a pas été accordée au praticien généraliste.

La position de la méthadone par jour a besoin de beaucoup de lignes et remplit du papier. Les remises sont enregistrées en général sur trois mois. Cette solution est nettement un recul face à la position «forfait par semaine» qui prévalait avant la version 1.05 .

Myra Wälti, Freidorf TG

\section{Réponse}

Chère Madame,

Les mises à jour du TARMED sont toujours effectuées avec effet au $1^{\text {er }}$ janvier de la nouvelle année; elles contiennent toutes les modifications intervenues dans la structure tarifaire du TARMED depuis la dernière version sur décision des partenaires tarifaires. Ces modifications sont plus ou moins nombreuses selon le groupe professionnel concerné. La révision du chapitre 30 (Imagerie médicale) est liée à des améliorations importantes de la structure tarifaire en radiologie pour les radiologues et les médecins de premier recours ainsi que dans le domaine des ultrasons pour les médecins de premier recours et les spécialistes. Concernant les délais extrêmement courts, j'ai fourni des informations détaillées à ce sujet dans le $n^{\circ}$ 4/2008 du BMS. De telles situations d'urgence ne devraient plus se présenter à l'avenir.

La facturation de la remise de méthadone correspond à une exigence des médecins de premier recours qui remettent de la méthadone; elle a donc été modifiée en conséquence.

Vous le voyez: il est très important de réviser la structure du TARMED car c'est le seul moyen d'en éliminer les erreurs. L'importance des répercussions que cela entraîne changent en fonction de la spécialisation concernée.

Dr Ernst Gähler, membre du Comité central de la FMH 